\title{
Nonlinear long-range plasmonic waveguides
}

\author{
Aloyse Degiron* and David R. Smith ${ }^{\dagger}$ \\ Center for Metamaterials and Integrated Plasmonics, Department of Electrical and Computer Engineering, \\ Duke University, Box 90291 Durham, North Carolina 27708, USA
}

(Received 26 July 2010; published 13 September 2010)

\begin{abstract}
We report on plasmonic waveguides made of a thin metal stripe surrounded on one or both sides by a Kerr nonlinear medium. Using an iterative numerical method, we investigate the stationary long-range plasmons that exist for self-focusing and self-defocusing Kerr-type nonlinearities. The solutions are similar to the wellknown case of infinitely wide nonlinear waveguides - they are strongly power-dependent and can experience symmetry-breaking bifurcations under appropriate conditions.
\end{abstract}

DOI: 10.1103/PhysRevA.82.033812

PACS number(s): 42.65.Wi, 73.20.Mf, 78.20.Bh

\section{INTRODUCTION}

Over the past decade, a variety of low-loss plasmonic components based on thin metal stripes has been demonstrated, including Bragg gratings [1], several couplers and interferometers [2-6], as well as thermo- and electro-optic modulators $[7,8]$. These structures can be designed and fabricated with a high degree of accuracy and may find useful applications as optical sensors or modulators [9]. To operate as low-loss waveguides, the metal stripes must be placed in a symmetric or slightly asymmetric environment. This condition ensures that the guided modes are long-range surface plasmons (SPs) characterized by a weak field confinement [10]. In practice, the symmetry requirements can be satisfied by burying the metallic structures in a dielectric host [1-8] or by patterning the stripes on ultrathin flexible membranes [11]. The surrounding media may also consist of nonlinear materials but the potential offered by this configuration remains largely unexplored for metal stripes of finite width.

While the number of studies on finite-width stripes embedded in nonlinear media is scant, there exists an abundant literature on nonlinear SPs guided by infinitely wide films [12-24]. Historically, the case of two-dimensional metal films bounded by materials exhibiting a power-dependent refractive index (Kerr nonlinearity) has occupied a central place in these investigations [14-20]. It was shown that the short- and longrange SPs that exist in the linear regime evolve into stationary nonlinear solutions, which are modes with properties that strongly depend on the power but that do not vary along the propagation direction $[15,16]$. In addition, a number of purely nonlinear stationary branches was also discovered, including nonintuitive SPs with a transverse electric polarization rather than the usual transverse magnetic polarization [17]. The properties of the stationary solutions result from the selfaction of their electromagnetic fields on the surrounding Kerr media. Despite serious mathematical difficulties, the field distribution and dispersion relation of these modes have been successfully described with increasingly rigorous analytical

\footnotetext{
*Present address: Univ. Paris-Sud and CNRS, Institut d'Electronique Fondamentale, UMR 8622, 91405 Orsay Cedex, France.

†drsmith@ee.duke.edu
}

theories [15-19]. Note that solving for the stationary modes is generally not sufficient for characterizing real nonlinear waveguides, if only because losses by absorption in the metal reduce the power carried by the modes as they propagate along the film. However, various numerical techniques are available to describe the evolution of real SPs from these solutions [24-28].

In this article, we extend the work done in two dimensions by solving for the stationary long-range SPs of thin metal stripes of finite width surrounded by various Kerr nonlinear media. As already shown for the purely linear case, SPs guided by the stripe geometry cannot be solved analytically because their electric and magnetic fields have nonzero components in all three directions that cannot be uncoupled in Maxwell's equations [10]. Here, we have computed the modes numerically with an iterative scheme in which a series of linear problems representing increasingly accurate descriptions of the system are solved using full-wave vectorial simulations. For each case considered in this study, we calculate the power dispersion relations that relate the wavevector of the modes with the power they carry. We discuss these results by comparing the computed curves with the evolution of the transverse electromagnetic field distribution of the modes.

Figure 1 shows the geometry considered in our study: A straight Au stripe (width $w=4 \mu \mathrm{m}$, thickness $t=50 \mathrm{~nm}$, and permittivity $\epsilon=-132+12.65 i)$ is sandwiched between a nonlinear cladding and a substrate which can be either linear or nonlinear. The calculations are performed at the telecommunication wavelength $\lambda=1.55 \mu \mathrm{m}$. The refractive index of the cladding varies with the electromagnetic power $I$ as $n_{c}=n_{0}+n_{2 c} I$, where $n_{0}=1.75$ and $n_{2 c}= \pm 10^{-9} \mathrm{~m}^{2} / \mathrm{W}$. The substrate is assumed to have either a constant index of refraction $n_{s}=n_{0}=1.75$ or the same material properties as the cladding $\left(n_{s}=n_{c}\right)$. Note that the values of $n_{2 c}$ have been taken from numerical studies investigating the stationary solutions of nonlinear dielectric waveguides [29-31], enabling a direct comparison with these structures. However, the nonlinear coefficients of real Kerr materials are usually much lower and exhibit saturation and anisotropy that are neglected in this article.

We write the electric field of the long-range SPs as $E=$ $E_{0}(x, y, P) \exp [\beta(P) . z]$, where $E_{0}$ is the transverse electric field, $\beta=\beta_{r}+i \beta_{i}$ is the complex wavevector, and $P$ is 


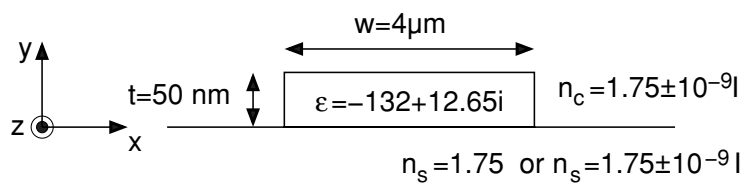

FIG. 1. Geometry of the nonlinear long-range plasmonic waveguide. The material parameters of the different regions summarize the different cases that will be considered in this study.

the total power carried by the mode. Since we focus on the stationary solutions, the problem can be formulated in two dimensions because $E_{0}$ and $\beta$ are invariant along the propagation direction $z$. The main difficulty in studying the structure shown in Fig. 1 is that the refractive index of the nonlinear region(s) has a gradient that depends itself on the solution. To address this problem, an iterative scheme must be applied to find the solutions at a given power $P$. The process starts by computing the transverse field pattern and wavevector of the linear long-range SP. For this task we use a commercial eigenmode solver based on the finite-element method (COMSOL Multiphysics). Next, we take the transverse power distribution $P_{\text {lin }}(x, y)$ of this solution to generate a nonlinear refractive index of the form $n_{0}+P n_{2 c} P_{\text {lin }}(x, y) / \iint P_{\text {lin }}(x, y) d x d y$. This index profile is entered into the mode solver and a new long-range SP is computed. At this stage the mode is not physically valid because its field distribution is different from the solution that has been used to define the nonlinear refractive index. Therefore the process must be reiterated until a self-consistent solution is found. We terminate the iterations when the field overlap integral between two consecutive passes approaches 1 with a residual of $10^{-5}$.

To better explore the space of possible solutions, we also used slightly modified versions of this simple iterative model. For example, we noticed that some simulations do not converge well at very high powers, in which case we seed the mode with a nonlinear solution obtained for a smaller value of $P$ rather than with the linear SP. In addition, certain configurations studied in this article admit two modes above a certain power threshold. To capture this behavior, it is necessary to pursue the iterations after the simulations have converged to the first mode because small numerical instabilities eventually cause an abrupt jump in the convergence process that lead to the second solution. It should be noted that variants of this iterative approach have already been widely used for studying the modes of three-dimensional nonlinear dielectric waveguides [29-33]. It is known to provide reliable solutions, even when multiple modes exist at the same power [30].

To validate our model, we have first simulated the nonlinear modes supported by an infinitely wide metal film and verified that the results are in quantitative agreement with the theoretical solutions supported by this geometry. An example of such comparisons between two-dimensional numerical simulations and theory will be shown later in this article.

\section{LINEAR SUBSTRATE AND SELF-DEFOCUSING CLADDING}

We first consider a metal stripe sandwiched between a linear substrate and a cladding with a self-defocusing nonlinearity

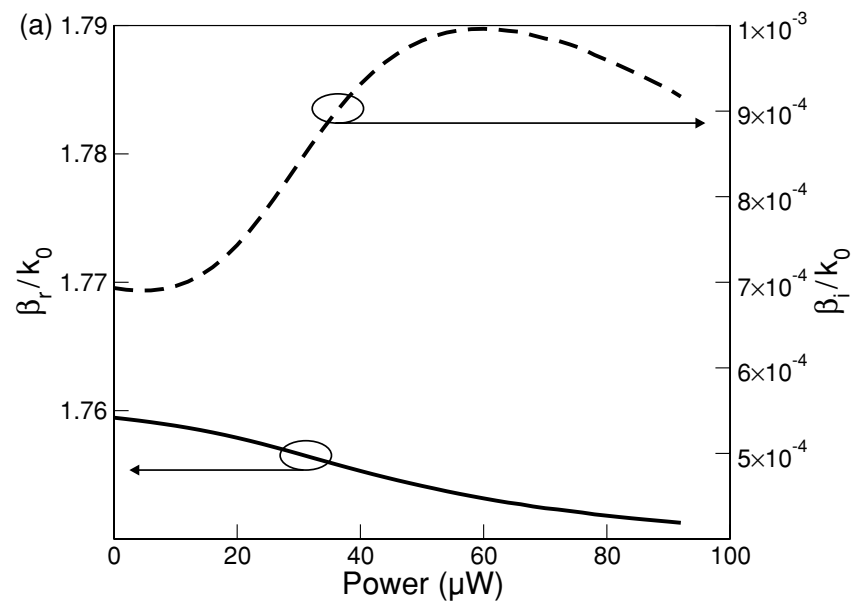

(b)

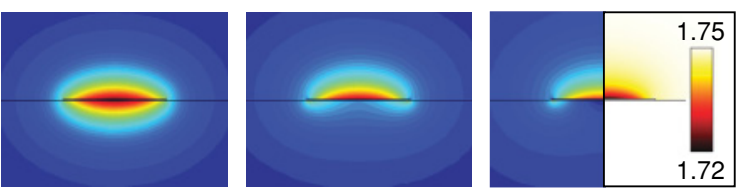

FIG. 2. (Color online) (a) Dispersion of the long-range SP supported by a metal stripe lying on a linear substrate and covered by a nonlinear cladding with $n_{2 c}=-10^{-9} \mathrm{~m}^{2} / \mathrm{W}$. The continuous curve corresponds to the evolution of $\beta_{r}$ while the dashed curve shows the dispersion of $\beta_{i}$. (b) Evolution of the $y$ component of the electric field at $P=0 \mu \mathrm{W}, P=40 \mu \mathrm{W}$, and $P=76 \mu \mathrm{W}$. Note that we have plotted the $y$ component of the field because its amplitude is orders of magnitude higher than the $x$ component [10]. For $P=76 \mu \mathrm{W}$, we have replaced the right half of the field pattern by the corresponding refractive index distribution.

$n_{2 c}=-10^{-9} \mathrm{~m}^{2} / \mathrm{W}$. Figure 2(a) shows the dispersion of the real and imaginary parts of the wavevector $\left(\beta_{r}\right.$ and $\beta_{i}$ ) with respect to the power carried by the mode. The curve representing the dispersion of $\beta_{r}$ has a negative slope and tends to a horizontal asymptote $\beta_{r}=1.75 k_{0}$, where $k_{0}=2 \pi / \lambda$. This value corresponds to the wavevector of a plane wave propagating in the linear substrate, suggesting that the long-range SP becomes unconfined at very high power levels. The evolution of $\beta_{i}$, which represents the attenuation of the mode, indicates that the transition from a guided mode to an unbound solution is not monotonic because the curve passes through a maximum at $P \approx$ $60 \mu \mathrm{W}$.

To gain insight into this behavior, we plot in Fig. 2(b) the transverse profile of the mode (the $y$ component of the electric field) at selected powers. The mode evolves from the symmetric, linear solution in the limit of weak fields into a more complex asymmetric pattern where the electric field is predominantly located in the lossless cladding. For the smallest powers, the asymmetric mode is more confined than the linear long-range SP, thereby increasing the losses by absorption in the metal. However, this trend is eventually inverted after the upturn in the dispersion of $\beta_{i}$. All these changes arise from the self-defocusing properties of the cladding, leading to smaller values of $n_{c}$ in the vicinity of the metal stripe as $P$ increases. An example of the refractive index distribution associated with a nonlinear SP is shown in the last plot of Fig. 2(b) for $P=$ $76 \mu \mathrm{W}$. 


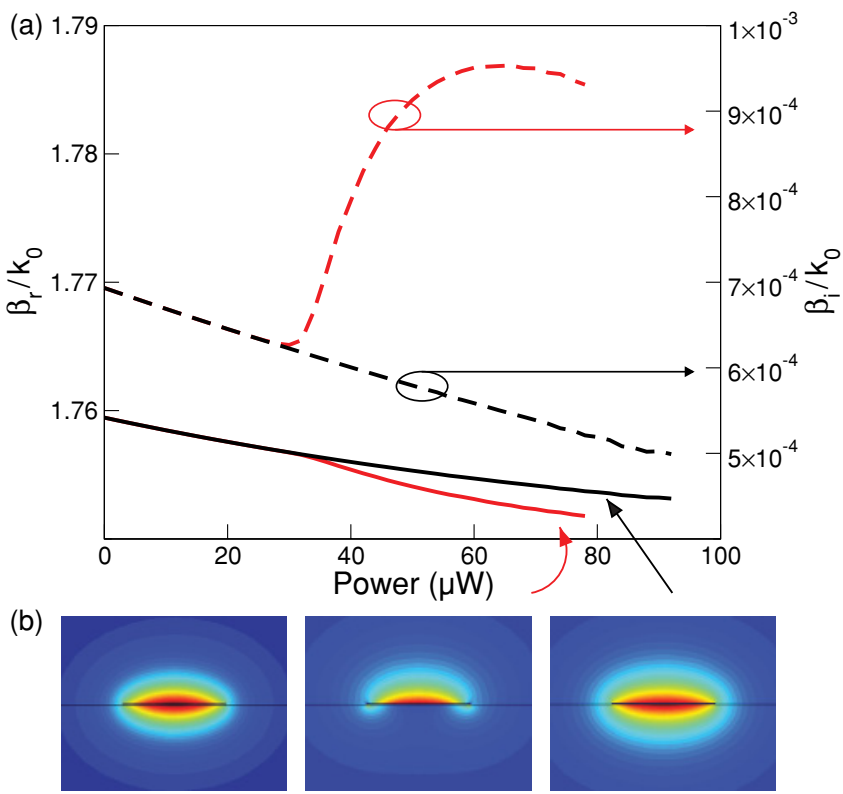

FIG. 3. (Color online) (a) Dispersion relation of the long-range SPs supported by a metal stripe surrounded on both sides by a nonlinear medium with $n_{2 c}=-10^{-9} \mathrm{~m}^{2} / \mathrm{W}$. The evolution of the symmetric and asymmetric modes is shown in black and red (gray), respectively. (b) Plots showing the $y$ component of the electric field before and after the bifurcation. From left to right: linear long-range SP at $P=0 \mu \mathrm{W}$, asymmetric branch at $P=78 \mu \mathrm{W}$, and symmetric branch at $P=80 \mu \mathrm{W}$.

Interestingly, the evolution of the nonlinear SP shown in Fig. 2 is very similar to the case of the infinitely wide metal film $[15,16]$. We shall see that the same remark is also true for the other configurations studied in this article.

\section{SELF-DEFOCUSING SUBSTRATE AND CLADDING}

We next study the case where both the substrate and the cladding have a self-defocusing nonlinearity $n_{2 c}=$ $-10^{-9} \mathrm{~m}^{2} / \mathrm{W}$. The corresponding power dispersion relations are plotted in Fig. 3(a). As in the previous case, the real part of the wavevector $\beta_{r}$ decreases with increasing $P$ because the refractive index around the stripe is depleted by the power carried by the mode. However, the curve splits into two branches at $P \approx 30 \mu \mathrm{W}$. The upper branch is characterized by a monotonic decrease of $\beta_{i}$ whereas the behavior of the lower branch is reminiscent of the evolution of the asymmetric nonlinear mode considered in Fig. 2.

The transverse field profiles before and after the symmetrybreaking bifurcation are shown in Fig. 3(b). The simulations indicate that the upper branch corresponds to a symmetric solution. Its field profile becomes gradually less confined but conserves the original symmetry of the linear mode, which is consistent with the monotonic behavior of both $\beta_{r}$ and $\beta_{i}$. The lower branch corresponds to an asymmetric solution having an electromagnetic field predominantly located in the cladding. In fact, this mode is degenerate and the same solution also exists with the field extending in the substrate. The field pattern of the asymmetric branch closely resembles the solution computed in Fig. 2, where only the upper cladding is nonlinear, so it is not surprising that its wavevector follows a similar evolution, with $\beta_{i}$ passing through a maximum at $P \approx 65 \mu \mathrm{W}$.

\section{LINEAR SUBSTRATE, SELF-FOCUSING CLADDING}

We now turn to self-focusing Kerr nonlinearities and compute the power dispersion relations for a linear substrate and a cladding with $n_{2 c}=+10^{-9} \mathrm{~m}^{2} / \mathrm{W}$. The results are summarized in Fig. 4(a). It can be seen that only one branch was found and that the curves representing $\beta_{r}$ and $\beta_{i}$ have a positive slope. This behavior is consistent with the fact that the refractive index of the cladding increases in the presence of the mode, improving its confinement but also increasing the losses by absorption in the metal.

This being said, the mode appears rather insensitive to the presence of the Kerr medium because the dispersion of $\beta_{r}$ is relatively flat. This result can be better understood by visualizing the transverse field pattern and refractive index distribution of the solutions [left and right insets of Fig. 4(a), respectively]. These plots reveal that the mode becomes asymmetric and that most of the signal propagates in the substrate. In other words, the interactions between the electric field and the nonlinear cladding are more limited than those
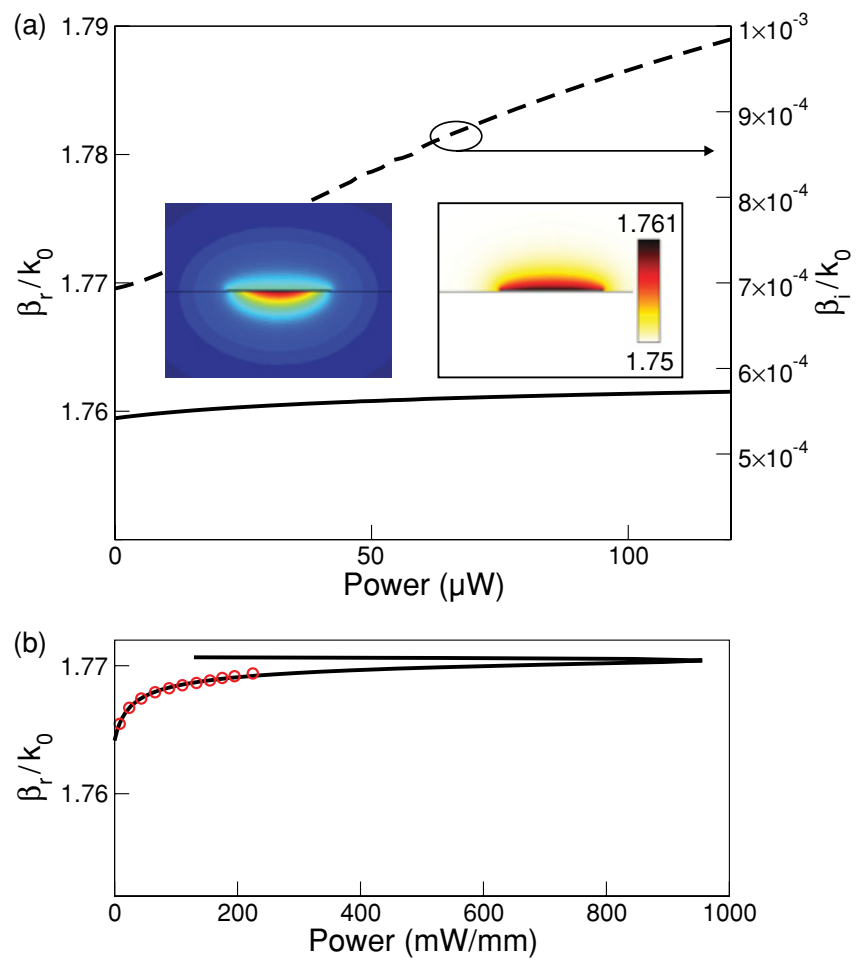

FIG. 4. (Color online) (a) Dispersion relation of the long-range SP supported by a metal stripe lying on a linear substrate and covered by a nonlinear cladding with $n_{2 c}=+10^{-9} \mathrm{~m}^{2} / \mathrm{W}$. The insets show the $y$ component of the electric field at $P=100 \mu \mathrm{W}$ (left) and the refractive index distribution at $P=100 \mu \mathrm{W}$ (right). (b) Dispersion relation in the case of an infinitely wide metal film with no material loss. All the other geometrical and material parameters are identical to those considered in (a). The continuous curve has been calculated using the theory developed in $[15,16]$ while the red open circles are the results of our numerical simulations. 
occurring in the case of self-defocusing Kerr materials, leading to smaller variations of the refractive index $n_{c}$.

It is instructive to compare the results of Fig. 4(a) with those obtained for a metal film of infinite width [Fig. 4(b)]. Here we have computed the solutions of this two-dimensional case using two different approaches: The continuous curve corresponds to the dispersion of $\beta_{r}$ predicted by the theory $[15,16]$, while the data points are the results of our numerical simulations. The theory predicts bistable solutions with a turning point at $P \approx 1000 \mathrm{~mW} / \mathrm{mm}$. The lower portion of the curve resembles the branch found for the three-dimensional case of Fig. 4(a). It is connected to an upper branch with a negative slope that abruptly goes toward smaller values of $P$.

Figure 4(b) indicates that our numerical simulations capture well the evolution of the lower branch but fail to predict the upper branch. In fact, this is a known limitation of the iterative solver adopted in this article. As is now well understood, branches with a positive slope are stable modes that do not change their character as the mode propagates. Conversely, branches with a negative slope correspond to a range of unstable solutions that quickly evolve into other guided and unguided modes $[25,26]$. These modes cannot be found with our iterative approach because it relies on a self-consistent process that only converges to stable solutions [30-32].

Based on these considerations, it is not impossible that the metal stripe of finite width investigated in Fig. 4(a) also exhibits a bistable behavior. This hypothesis is further reinforced by the fact that bistability arises frequently with optical waveguides bound by a linear substrate and a selffocusing cladding (see, e.g., [30-32]). Further work is required to elucidate this question. As proposed in [31] for nonlinear dielectric waveguides, it should be possible to find the unstable solutions by reformulating the system of differential equations solved at each iteration in such a way that its eigenvalue is the square root of $P$ instead of the wavevector $\beta$.

\section{SELF-FOCUSING SUBSTRATE AND CLADDING}

Finally, we consider a metallic stripe surrounded on both sides by self-focusing nonlinear media $\left(n_{2 c}=+10^{-9} \mathrm{~m}^{2} / \mathrm{W}\right)$. The transverse field profiles shown in Fig. 5 reveal that the mode remains rigorously symmetric but increasingly confined. The dispersion of the mode is therefore monotonic, with both $\beta_{r}$ and $\beta_{i}$ indefinitely increasing as $P \rightarrow \infty$. In addition, $n_{c}$ and $n_{s}$ quickly tend to arbitrarily high values-for $P \approx$ $100 \mu \mathrm{W}$, for example, the changes in the refractive index are six times larger than those occurring when only the cladding is nonlinear [compare the last plot of Fig. 5(b) to the right inset of Fig. 4(a)]. Note however that in real Kerr materials, the saturation of the nonlinear index prevents $n_{c}$ and $n_{s}$ from diverging.

These results are once again very similar to the case of an infinitely wide metal film bound by the same nonlinear media. However, the two-dimensional film admits another branch in addition to the symmetric one $[15,16]$. This additional solution corresponds to a degenerate mode with a transverse field distribution having a maximum in one of the nonlinear media. The asymmetric solution is a purely nonlinear mode with a cutoff at low power. It has an evolution similar to that of the symmetric SP, except that it can only occur above a certain

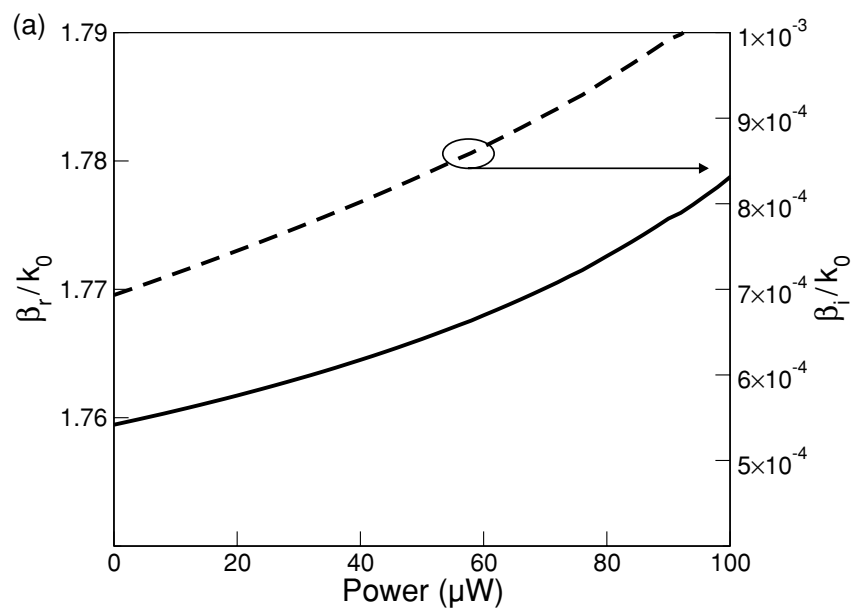

(b)
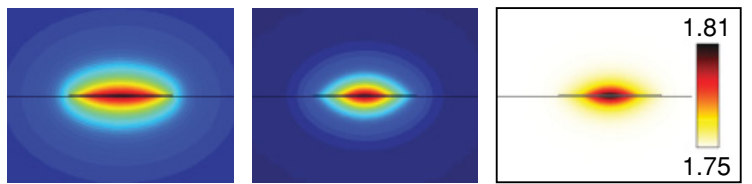

FIG. 5. (Color online) (a) Dispersion relation of the long-range SP supported by a metal stripe surrounded on both sides by a nonlinear medium with $n_{2 c}=+10^{-9} \mathrm{~m}^{2} / \mathrm{W}$. (b) $y$ component of the electric field at $P=0 \mu \mathrm{W}$ (left) and $P=98 \mu \mathrm{W}$ (middle), and refractive index distribution of the mode at $P=98 \mu \mathrm{W}$ (right).

power threshold above which a self-focusing channel is created in the nonlinear medium.

We have not been able to find a purely nonlinear asymmetric solution for the metal stripe of finite width. However, this negative result does not necessarily imply that an asymmetric mode cannot be sustained. It should be noted that all the modes must be seeded properly so one cannot exclude that the asymmetric nonlinear solution requires a starting point that has not been considered in this study.

\section{CONCLUSION}

We have investigated the stationary long-range SPs sustained by a metal stripe of finite width surrounded by different combinations of linear and Kerr nonlinear media. To solve for the modes, we have used an iterative numerical technique that has already been successfully implemented in the past to characterize nonlinear dielectric waveguides. This method is very flexible and can be readily adapted to study other plasmonic geometries as well. The main drawback is that it requires an initial guess to converge to the different nonlinear modes. A final comment should be made about the powers required to observe the effects shown in Figs. 2-5. We have seen that significant changes occur for powers smaller than $100 \mu \mathrm{W}$-interestingly, the wavevector shifts are comparable to those predicted for dielectric waveguides with the same levels of nonlinearity [29-31]. Nevertheless, it will be difficult to directly observe these modes using continuous wave excitation because real Kerr materials have nonlinear coefficients that are usually much smaller than those considered here. Further developments are thus needed to study the propagation of pulsed SPs with high peak power densities along these structures. 
[1] S. Jetté-Charbonneau, R. Charbonneau, N. Lahoud, G. A. Mattiussi, and P. Berini, IEEE J. Quantum Electron. 41, 1480 (2005).

[2] A. Boltasseva, T. Nikolajsen, K. Leosson, K. Kjaer, M. S. Larsen, and S. I. Bozhevolnyi, J. Lightwave Technol. 23, 413 (2005).

[3] H. S. Won, K. C. Kim, S. H. Song, C.-H. Oh, P. S. Kim, S. Park, and S. I. Kim, Appl. Phys. Lett. 88, 011110 (2006).

[4] R. Charbonneau, C. Scales, I. Breukelaar, S. Fafard, N. Lahoud, G. Mattiussi, and P. Berini, J. Lightwave Technol. 24, 477 (2006).

[5] A. Degiron, S.-Y. Cho, T. Tyler, N. M. Jokerst, and D. R. Smith, New J. Phys. 11, 015002 (2009).

[6] Y.-J. Tsai, A. Degiron, N. M. Jokerst, and D. R. Smith, Opt. Express 17, 17471 (2009).

[7] T. Nikolajsen, K. Leosson, and S. I. Bozhevolnyi, Appl. Phys. Lett. 85, 5833 (2004).

[8] P. Berini, R. Charbonneau, S. Jetté-Charbonneau, N. Lahoud, and G. Mattiussi, J. Appl. Phys. 101, 113114 (2007).

[9] P. Berini, Adv. Opt. Photon. 1, 484 (2009).

[10] P. Berini, Phys. Rev. B 61, 10484 (2000).

[11] P. Berini, R. Charbonneau, and N. Lahoud, Nano Lett. 7, 1376 (2007).

[12] R. T. Deck and D. Sarid, J. Opt. Soc. Am. 72, 1613 (1982).

[13] J. C. Quail, J. G. Rako, H. J. Simon, and R. T. Deck, Phys. Rev. Lett. 50, 1987 (1983).

[14] G. I. Stegeman, J. J. Burke, and D. G. Hall, Appl. Phys. Lett. 41, 906 (1982).

[15] G. I. Stegeman and C. T. Seaton, Opt. Lett. 9, 235 (1984).

[16] J. Ariyasu, C. T. Seaton, G. I. Stegeman, A. A. Maradudin, and R. F. Wallis, J. Appl. Phys. 58, 2460 (1985).
[17] G. I. Stegeman, J. D. Valera, C. T. Seaton, J. Sipe, and A. A. Maradudin, Solid State Commun. 52, 293 (1984).

[18] K. M. Leung, Phys. Rev. B 32, 5093 (1985).

[19] A. D. Boardman, A. A. Maradudin, G. I. Stegeman, T. Twardowski, and E. M. Wright, Phys. Rev. A 35, 1159 (1987).

[20] R. K. Hickernell and D. Sarid, J. Opt. Soc. Am. B 3, 1059 (1986).

[21] A. R. Davoyan, I. V. Shadrivov, and Y. S. Kivshar, Opt. Express 16, 21209 (2008).

[22] H. Yin, C. Xu, and P. M. Hui, Appl. Phys. Lett. 94, 221102 (2009).

[23] W. Shao, L. Li, W. Liu, T. Zhang, H. Ma, J. Xu, and J. Tian, Appl. Phys. Lett. 95, 211105 (2009).

[24] Y.-Y. Lin, R.-K. Lee, and Y. S. Kivshar, Opt. Lett. 34, 2982 (2009).

[25] J. V. Moloney, J. Ariyasu, C. T. Seaton, and G. I. Stegeman, Appl. Phys. Lett. 48, 826 (1986).

[26] L. Leine, C. Wächter, U. Langbein, and F. Lederer, J. Opt. Soc. Am. B 5, 547 (1988).

[27] K. Hayata, A. Misawa, and M. Koshiba, J. Opt. Soc. Am. B 7, 1772 (1990).

[28] D. Mihalache, D.-M. Baboiu, and D. Mazilu, Opt. Commun. 110, 67 (1994).

[29] K. Hayata, M. Nagai, and M. Koshiba, IEEE Trans. Microwave Theory Tech. 36, 1207 (1988).

[30] K. Hayata and M. Koshiba, J. Opt. Soc. Am. B 5, 2494 (1988).

[31] Q. Y. Li, R. A. Sammut, and C. Pask, Opt. Commun. 94, 37 (1992).

[32] X. H. Wang and G. K. Cambrell, J. Opt. Soc. Am. B 10, 1090 (1993).

[33] S. R. Cvetkovic, A. P. Zhao, and M. Punjani, Microwave Opt. Technol. Lett. 7, 293 (1994). 